\title{
Feasibility, tolerability, and efficacy of the
} concurrent addition of erlotinib to thoracic radiotherapy in locally advanced unresectable non-small-cell lung cancer: a Phase II trial

\author{
This article was published in the following Dove Press journal: \\ OncoTargets and Therapy \\ I March 2016 \\ Number of times this article has been viewed
}

\section{Enrique Martínez' \\ Maite Martínez' \\ Mikel Rico' \\ Berta Hernández' \\ Francesc Casas ${ }^{2}$ \\ Nuria Viñolas ${ }^{2}$ \\ Ana Pérez-Casas ${ }^{3}$ \\ Manuel Dómine ${ }^{3}$ \\ Julián Mínguez ${ }^{4}$}

'Radiation Oncology Services, Hospital of Navarra, Pamplona, Spain; ${ }^{2}$ Medical Oncology Department, Clinical Hospital of Barcelona, Barcelona, Spain; ${ }^{3}$ Department of Radiation Oncology, Jiménez Díaz Foundation, Madrid, Spain; ${ }^{4}$ Department of Radiation Oncology, University Hospital of Donostia, San Sebastian, Spain
Correspondence: Enrique Martínez Radiation Oncology Services, Hospital of Navarra, Calle Irunlarrea, 3, 31008 Pamplona, Navarra, Spain

Tel +34948255400

Fax +34948296500

Email enrique.martinez.lopez@cfnavarra.es
Purpose: Although many studies have confirmed the synergic effects of combining chemotherapy (CT) and radiotherapy (RT), clinical data evaluating safety and efficacy of erlotinib in combination with RT in locally advanced non-small-cell lung cancer (NSCLC) are limited. The aim of this study was to determine the feasibility, tolerability, and efficacy of the concurrent addition of erlotinib to the standard three-dimensional conformal thoracic RT in patients with unresectable or locally advanced NSCLC who are not candidates for receiving standard CT.

Patients and methods: Feasibility and tolerability, assessed by evaluating adverse events (AEs), and effectiveness, by calculating progression-free survival (PFS), overall survival (OS), cancer-specific survival (CSS), and objective response rate (ORR), were analyzed in 30 patients receiving RT alone and 60 receiving RT and erlotinib.

Results: Erlotinib with RT showed an extended CSS and a higher rate of complete responses compared with RT alone. No differences between groups were found regarding OS, PFS, and ORR. AEs were significantly higher in the combined treatment, which mainly included cutaneous toxicity, dyspnea, fatigue, hyporexia, diarrhea, and infection. Erlotinib did not increase the toxicity produced by RT.

Conclusion: The combination of erlotinib with RT produced, in our study, a scarce clinical benefit in the treatment of unresectable or locally advanced NSCLC, limited to complete responses and longer CSS rate compared with RT alone. Increased toxicity events were associated with combined therapy, which mainly included cutaneous toxicity. In our opinion, further studies in molecularly unselected lung cancer patients treated with EGFR TKIs and RT are not indicated. The use of biomarkers for the identification of patients that are most likely to benefit from this treatment is an essential next step in the research of this condition.

Keywords: non-small-cell lung cancer, radiotherapy, erlotinib, concurrent

\section{Introduction}

Non-small-cell lung cancer (NSCLC) represents the most common type of lung cancer $(80 \%-85 \%)$ and is one of the leading causes of cancer-related mortality worldwide. ${ }^{1,2}$ Overall 5-year survival rate is $\sim 15 \%$, but depending on the stage of the disease ranges from $73 \%$, in stage IA, to $2 \%$, in stage IV. ${ }^{3}$ The majority of patients present with unresectable disease, and $\sim 40 \%$ of them in advanced stages. ${ }^{4,5}$ In the last decades, the standard treatment for unresectable or locally advanced NSCLC has varied from single thoracic radiotherapy (RT) to its combination with chemotherapy (CT), ie, chemoradiotherapy. ${ }^{6-8}$ Several trials have shown the higher efficiency, in disease 
control and overall survival (OS), of combining both RT and CT with respect to RT alone. ${ }^{9-11}$ Moreover, their concurrent administration has been demonstrated to achieve even better OS rates than the sequential scheme. ${ }^{12-14}$ However, such concurrence of therapies has been associated with higher incidence of grade 3-4 adverse events (AEs), due to increased toxicities. Novel therapeutic agents have been designed to specifically target molecular alterations occurring in NSCLC. Drugs targeting the inhibition of growth factor receptor signaling or angiogenesis have been achieving good clinical outcomes. ${ }^{15-17}$ Erlotinib is an inhibitor of the tyrosine kinase (TKI) activity of the epidermal growth factor receptor (EGFR) that has been approved for patients with advanced NSCLC who failed at least one prior CT regimen. Erlotinib has also been proven to show significant clinical activity in patients with tumors harboring EGFR mutation. ${ }^{18}$ Although many studies have confirmed the synergic effects of combining CT and RT, ${ }^{6}$ clinical data evaluating safety and efficacy of erlotinib in combination with RT in advanced NSCLC are currently limited. ${ }^{19}$

Therefore, the objective of this study was to determine the feasibility, tolerability, and efficacy of the concurrent addition of erlotinib to the standard three-dimensional conformal thoracic RT treatment in patients with unresectable or locally advanced NSCLC.

\section{Patients and methods}

This multicenter, randomized, controlled, open-label, prospective, and nationwide Phase II trial involved patients with unresectable or locally advanced NSCLC. Study design, endpoints, and study assessment are summarized in Table S1.

Eligibility criteria: The criteria for inclusion in the study were: aged over 18 years; histologically confirmed stage IA-IIIB unresectable NSCLC; not susceptible for receiving standard CT; measurable disease; Eastern Cooperative Oncology Group Performance Status (ECOG-PS) 0-2; adequate hematologic, hepatic, renal, and respiratory function; and no previous CT or RT treatment. The main exclusion criteria were: previous $\mathrm{CT}$ or RT treatment; previous treatment with erlotinib or any anti-EGFR; any concomitant severe disorder or neoplasia; and pregnancy.

Study design: The control arm (CA) consisted of patients receiving 66 Gy with three-dimensional conformal thoracic RT (5 days/week, conventional fractionation 200 cGy/day), whereas the experimental arm (EA) consisted of patients receiving the same $\mathrm{RT}$ dose scheme but combined with erlotinib (150 mg per os [po] for a maximum of 6 months). The rules for tumor delineation in this study were common to all the participants' hospitals following accepted standard in three-dimensional panning. ${ }^{20}$ Randomization was centralized, and patients were stratified according to ECOG-PS ( $0-1$ versus 2$)$ and stage of disease (I-II versus III). Primary endpoint was to determine the feasibility and tolerability of the addition of erlotinib to thoracic RT in these patients. The secondary endpoint was to assess the efficacy of the combined treatment, including progression-free survival (PFS), time to progression (TTP), time to treatment failure (TTF), OS, cancer-specific survival (CSS), and objective response rate (ORR). On the basis of primary endpoint and the lack of information concerning the combination of erlotinib and RT, the total number of patients enrolled in the study was 90, ie, 30 in CA and 60 in EA. All patients gave their written informed consent before participating in the study. Procedures were performed in accordance with the guidelines established by the ethics committee of each participating center and the Declaration of Helsinki. This study was approved by the ethics committees of the Hospital of Navarra, Clinical Hospital of Barcelona, Jiménez Díaz Foundation, and University Hospital of Donostia.

\section{Patient evaluation}

Total follow-up was calculated from randomization, in March 2006, to the last follow-up visit, in December 2011. Feasibility and tolerability were determined by evaluating the percentage of patients developing grade 3-4 AEs, according to National Cancer Institute-Common Terminology Criteria for Adverse Events NCI-CTCAE v3.0. Complete response (CR), partial response (PR), stable disease (SD), and progressive disease (PD) were assessed using Response Evaluation Criteria in Solid Tumours (RECIST) criteria. No response was defined when PD was observed before tenth week (during RT or just afterward). ORR was calculated as the percentage of patients achieving a CR or PR. Feasibility and tolerability were determined by evaluating the percentage of patients developing grade 3-4 AEs, according to NCI-CTCAE v3.0.

\section{Statistical analysis}

Comparisons between groups were assessed using Fisher's exact test. Survival functions were estimated using KaplanMeier method (95\% confidence interval [95\% CI]). A logrank test was used to compare survival functions between experimental groups. Hazard ratio (HR) was calculated by using the Cox regression model. TTF was calculated as the time from randomization to PD or to treatment discontinuation by any cause. OS was calculated as the time between 
randomization to death, or alive (censored), and PFS to disease progression or death, or censored. The CSS was calculated as the time from randomization to death due to PD. Statistical significance was established when $P \leq 0.05$. All the statistical procedures were performed using SAS 9.2 and posteriors.

\section{Results}

\section{Patients and tumors at baseline}

Demographic and clinical characteristics of patients at baseline are shown in Table S2. All the patients from the CA $(\mathrm{N}=30)$ and $90.0 \%$ from the EA were male. The median age was 79.8 (interquartile range [IQR]: 74.6-81.2) and 79.3 (IQR: 76.7-81.7) years for CA and EA, respectively. In the CA, approximately half of the patients were smokers $(46.7 \%)$ and the other half ex-smokers (50.0\%). In the EA, 6.7\% were nonsmokers, $21.7 \%$ smokers, and $71.7 \%$ ex-smokers. History of interstitial lung disease was reported in $3.3 \%$ and $6.7 \%$ of patients from CA and EA, respectively. Most of the patients (60.0\% in CA and 63.3\% in EA) had ECOG-PS 1 at baseline, while $40.0 \%$ and $31.7 \%$ had ECOG-PS 2, respectively. The most prevalent tumor stage was IIIB (40.0\% versus $38.3 \%$ in CA and EA, respectively), followed by IB (20.0\% versus $18.3 \%$ ), IIIA (13.3\% versus $16.7 \%)$, and IA ( $13.3 \%$ versus $11.7 \%$ ). Squamous-cell carcinoma was detected in $46.7 \%$ of the patients from each group.

The median of treatment duration with RT was 7 weeks. The median total cumulative dose was 66 Gy. Erlotinib was combined concurrently with RT in the EA for a median of 1.8 months (IQR: 1.6-1.8). The median total cumulative dose of erlotinib was $6,850 \mathrm{mg}$ (IQR: 4,825-7,500). The median dose intensity of erlotinib was $0.98 \mathrm{mg}$ (IQR: 0.72-1.00). Erlotinib was maintained in monotherapy after RT in EA for a maximum of 6 months. The median total duration with erlotinib in monotherapy was 17.1 weeks (IQR: 4.1-19.2). The median total follow-up was 15.2 (IQR: 6.3-20.8) and 11.7 months (IQR: 5.7-22.6) in CA and EA, respectively.

\section{Efficacy outcome}

The response of tumor to the treatment was evaluated in 78 patients, 28 from the CA $(93.3 \%)$ and 50 from the EA (83.3\%). These responses are summarized in Table 1. CR was achieved in $21.4 \%$ versus $41.5 \%$ of CA and EA, respectively; PR in $57.1 \%$ versus $32.1 \%$; SD in $14.3 \%$ versus $11.3 \%$; PD in $3.6 \%$ versus $15.1 \%$. Only one patient from the CA $(3.6 \%)$ showed no response to the treatment. These differences in response rate were statistically significant $(P=0.046)$ between
Table I Responses of tumor to treatment arms

\begin{tabular}{lll}
\hline Responses & $\begin{array}{l}\mathbf{R T} \text { arm } \\
(\mathbf{N}=\mathbf{2 8})\end{array}$ & $\begin{array}{l}\mathbf{R T}+\text { erlotinib arm } \\
\mathbf{( N = 5 3 )}\end{array}$ \\
\hline $\begin{array}{lll}\text { Tumor responses, n (\%) } \\
\text { CR }\end{array}$ & $6(21.4)$ & $22(41.5)$ \\
PR & $16(57.1)$ & $17(32.1)$ \\
SD & $4(14.3)$ & $6(11.3)$ \\
PD & $1(3.6)$ & $8(15.1)$ \\
No response & $1(3.6)$ & $0(0.0)$ \\
ORR, n (\%) & $22(78.6)$ & $39(73.6)$ \\
\hline
\end{tabular}

Abbreviations: $R T$, radiotherapy; $C R$, complete response; PR, partial response; $\mathrm{SD}$, stable disease; $\mathrm{PD}$, progressive disease; $\mathrm{ORR}$, objective response rate.

both the arms. ORR was achieved in $78.6 \%$ of the CA and in $73.6 \%$ of the EA. The median PFS was 11.4 months $(95 \%$ CI, 7.5-13.5) in the CA and 8.9 months (95\% CI, 6.8-12.9) in the EA (Figure 1). No significant differences were found between both the groups (HR 1.05; 95\% CI, 0.66-1.68; $P=0.835$ ). When considering the type of tumor response, patients from the CA who achieved a CR had a PFS (22.1 months; 95\% CI, 2.4-71.4) significantly higher (HR 0.29; 95\% CI, 0.08-1.03; $P=0.044)$ than patients achieving the remaining of tumor responses (11.4 months; 95\% CI, 7.513.4). Although patients from the EA with $C R$ had a higher PFS (15.3 months; 95\% CI, 10.8-19.5) than the remaining patients (8.0 months; 95\% CI, 6.8-12.9), this difference was not statistically significant (HR 0.60; 95\% CI, 0.31-1.18; $P=0.134)$. The multivariate analysis showed that achieving a CR influenced the PFS rate (HR 0.94; 95\% CI, 0.28-0.88; $P=0.017$ ). TTF was not significantly different (HR $1.49 ; 95 \%$ CI, 0.93-2.38; $P=0.092)$ between the CA (11.4 months; $95 \%$ CI, 7.5-13.5) and EA (5.2 months; 95\% CI, 3.0-7.3). The median OS was 15.3 months (95\% CI, 10.1-20.8) in the CA and 12.9 months (95\% CI, 8.0-17.2) in the EA. No significant differences were found between both groups (HR 1.18; 95\% CI, 0.73-1.92; $P=0.493$ ). CSS was not significant (HR $0.86 ; 95 \%$ CI, $0.46-1.61 ; P=0.645)$ between the CA $(17.7$ months; 95\% CI, 11.7-34.7) and EA (21.4 months; 95\% CI, 15.0-33.1) (Figure 2).

\section{Safety outcome}

The radiation dose was modified in $20.7 \%$ of patients from the $\mathrm{CA}$ and in $33.3 \%$ from the EA. Regarding erlotinib, $26.7 \%$ of patients required treatment interruption and $21.7 \%$ dose reduction. The main reasons for modifying the dose of erlotinib were: developing an AE (53.1\%), improvement from an $\mathrm{AE}(37.5 \%)$, and others (9.4\%). Among the 51 patients continuing with erlotinib monotherapy, $27.5 \%$ required treatment interruption, $19.6 \%$ dose reduction, and 


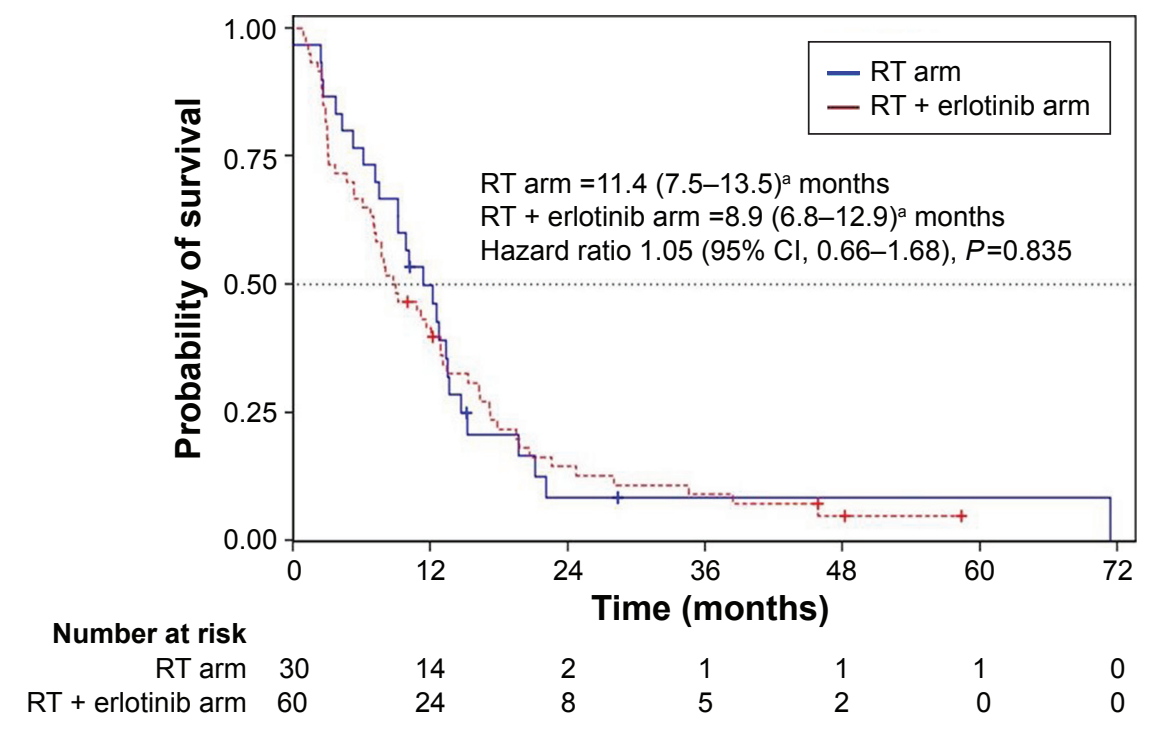

Figure I Overall survival for each treatment arm.

Note: a Data presented as median (range).

Abbreviations: $\mathrm{RT}$, radiotherapy; $\mathrm{Cl}$, confidence interval.

$17.7 \%$ dose escalation. The treatment (RT) was completed in $70.0 \%$ of patients from the CA and in $40.0 \%$ (whole treatment) from the EA. The reasons for interrupting the treatment were: unacceptable toxicity $(0.0 \%$ versus $18.3 \%$, respectively), violation of protocol $(0.0 \%$ versus $2.3 \%)$, consent withdrawal $(0.0 \%$ versus $8.3 \%)$, investigator decision $(0.0 \%$ versus $8.3 \%)$, PD ( $13.3 \%$ versus $8.3 \%)$, or death (16.7\% versus $13.3 \%)$. Main AEs developed during the treatment are shown and explained in Table S3.

Cutaneous toxicity was the most frequent $\mathrm{AE}(66.7 \%$ of patients from CA and $86.7 \%$ from EA developed at least one cutaneous event). Cutaneous events included acneiform rash, desquamation, dermatitis, eczema, folliculitis, and radiation skin injury. Grade $\geq 3 \mathrm{rash} /$ desquamation was found in $0.0 \%$ versus $13.3 \%$ in CA and EA, respectively; radiation skin injury in $3.4 \%$ versus $3.3 \%$; and dry skin in $0.0 \%$ versus $1.7 \%$. The incidence of developing cutaneous rash was not significantly associated with achieving, or not achieving, a complete tumor response or with ORR. When considering the incidence of cutaneous rash, patients from the EA who developed cutaneous rash had a PFS (11.1 months; 95\% CI, 7.7-15.3) significantly higher $(P<0.0001)$ than

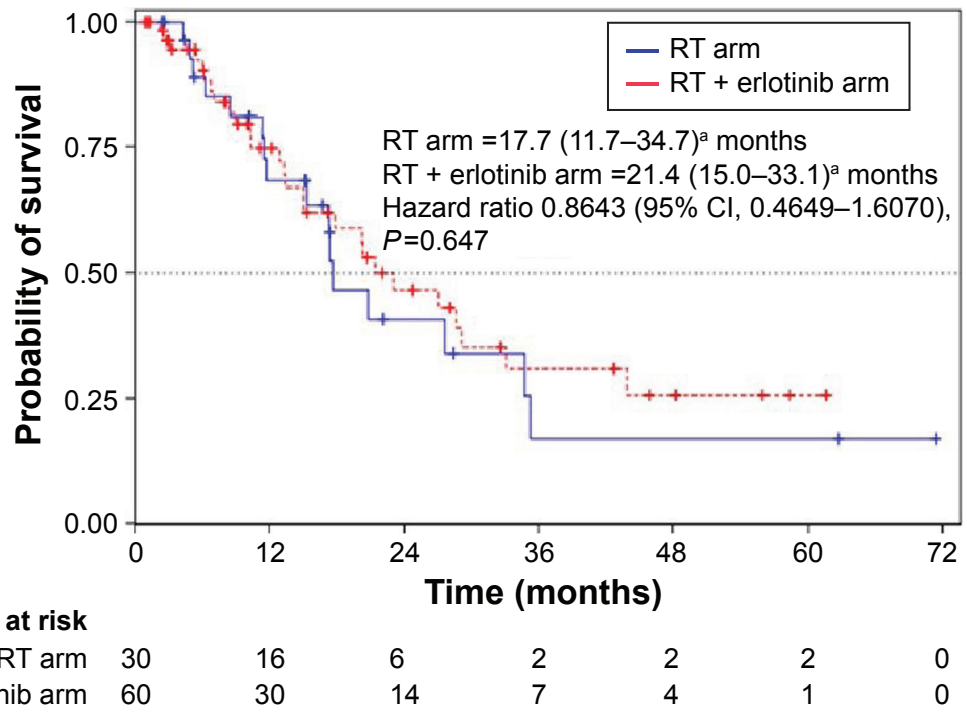

Figure 2 Cancer-specific survival for each treatment arm. Note: a Data presented as median (range).

Abbreviations: $\mathrm{RT}$, radiotherapy; $\mathrm{Cl}$, confidence interval. 
the patients who did not develop it (2.8 months; 95\% CI, 1.6-7.3). The multivariate analysis showed that developing cutaneous rash influenced the PFS rate (HR 0.52; 95\% CI, $0.28-0.98 ; P=0.043)$. The association of erlotinib with RT did not increase the cutaneous damage within the irradiation field. The main grade 3-5 AEs reported were the following: dyspnea (13.8\% versus $10.0 \%$ in CA and EA, respectively), fatigue $(0.0 \%$ versus $8.3 \%)$, hyporexia $(3.4 \%$ versus $8.3 \%)$, diarrhea $(3.4 \%$ versus $6.7 \%)$, and infection $(3.4 \%$ versus $6.7 \%)$. The number of patients with at least one severe $\mathrm{AE}$ was significantly higher $(P=0.045)$ in the EA $(50.0 \%)$ when compared to the CA $(27.6 \%)$. The number of patients with grade 3-5 AEs was significantly higher $(P=0.016)$ in the EA (65.0\%) compared to the CA (37.9\%). Only $21.7 \%$ of patients from the EA developed severe AEs caused directly by erlotinib, five patients developed pneumonitis grade $3-5$, three (10\%) in the CA and two $(3.2 \%)$ in the EA, but the only patient who died by this cause belonged to the EA. Main AEs observed in the four patients from the EA with history of interstitial lung disease were grade 1-2 fatigue, rash/desquamation, and diarrhea (three patients each), but none presented pneumonitis as AE.

\section{Discussion}

RT in combination with CT is the standard for treatment of unresectable or locally advanced NSCLC. Clinical data concerning safety and efficacy of erlotinib when combined concurrently with thoracic RT are currently scarce. Results from our study showed no differences regarding OS, PFS, and ORR. However, erlotinib in combination with RT showed an extended CSS and a higher rate of complete responses, compared with RT alone. By contrast, AEs were significantly higher in the combined treatment, which mainly included cutaneous toxicity.

The combination of RT and CT has been demonstrated to achieve better clinical outcomes, in regard to control of disease and survival rates than RT alone. ${ }^{9-11}$ In 1995, a metaanalysis evaluating data from eleven randomized clinical trials demonstrated that cisplatin-based regimes combined with RT led to a $13 \%$ decreased risk of death than RT alone. ${ }^{21}$ Similarly, in 2010, an updated meta-analysis of data from 19 trials showed that the addition of CT to RT decreased the overall risk of death (HR 0.71; 95\% CI, 0.64-0.80) and extended the overall PFS (HR 0.69; 95\% CI, 0.58-0.81). ${ }^{13}$ Furthermore, a meta-analysis in 2011 compared the effectiveness of concurrent and sequential chemoradiotherapy regimens, and the results indicated that the concurrent scheme improved OS (HR 0.84; 95\% CI, 0.74-0.95), presenting an absolute benefit of $5.7 \%$ at 3 years and $4.5 \%$ at 5 years. ${ }^{12}$ Concomitant to this benefit, the experimental groups with concurrent administration also showed higher incidence of severe AEs, mainly esophageal toxicity.

The efficacy of erlotinib in combination with RT has been extensively evaluated in clinical studies involving patients with different cancer types; ${ }^{19,22-24}$ however, few randomized clinical studies have been performed for NSCLC. ${ }^{25}$ Indeed, there is no clinical trial to date concerning the safety and effectiveness of erlotinib when administered concurrently with RT. Results from our study showed an extended CSS for the combined therapy (21.4 months) compared with RT alone (17.7 months; not statistically significant), and also a higher rate of complete responses for the combined treatment (41.5\%) compared with RT alone (21.4\%; statistically significant). Furthermore, patients achieving CR showed higher PFS than other patients (22.1 months in RT alone and 15.3 months in the combined treatment). Additional results from the study showed no differences between groups with respect to OS (15.3 versus 12.9 months in RT alone and the combined treatment, respectively), PFS (11.4 versus 8.9 months), and ORR (78.6\% versus 73.6\%). Results obtained for the combination therapy of erlotinib with RT were higher than those reported for erlotinib as first-line monotherapy in EGFR-unselected patients. ${ }^{18,19}$ Nevertheless, they are more in accordance with the values reported for patients harboring activating EGFR mutation. ${ }^{18}$ A main limitation of the study was the lack of information concerning EGFR mutation from the patients. Although EGFR mutation status has been demonstrated to influence therapeutic results, at the time of our study design, the information published in the literature was not conclusive enough to make the determination of the mutation status in patients mandatory.

In our study, RT alone achieved better clinical outcomes (but not statistically significant) than the concurrent administration with erlotinib. This result might be explained by the characteristics of patients at baseline, ie, with ECOG-PS 2 (31.7\% of patients), with tumor stage IIIB (38.3\%), with smoking habits (21.7\% smokers and $71.7 \%$ ex-smokers), and a median age of 79.3 years. The combination of these poor prognosis factors (elderly population, advanced tumor stages, and poor ECOG-PS) might have produced a negative influence on the effectiveness of erlotinib. Some elderly patients with NSCLC are not candidates for CT due to the risk of developing comorbidities. ${ }^{26}$ For this reason, RT is recommended to be administered alone in patients with locally advanced unresectable tumor who are not candidates for CT or in palliative treatment. These results are consistent with other disappointing 
results regarding the lack of benefit when combining cetuximab or erlotinib/gefitinib with RT, such as RTOG 0617, CALGB 30605, and CALGB 30106, in lung cancers.

An increased number of AEs were reported in the combined treatment. In this treatment arm, the main AE developed was cutaneous toxicity (observed in $86.7 \%$ of patients). However, erlotinib did not increase the cutaneous damage caused by RT within the irradiation field, and caused neither pulmonary nor esophageal toxicity.

Main grade 3-5 AEs that were noticed were dyspnea $(10.0 \%)$, fatigue $(8.3 \%)$, hyporexia $(8.3 \%)$, diarrhea $(6.7 \%)$, and infection (6.7\%). They were not different from the published ones in other studies with erlotinib. It is interesting to note that in the erlotinib group, the incidence of cutaneous rash was associated with a significant longer PFS (11.1 months), compared with the group having no cutaneous rash (2.8 months). This result is in accordance with previous literature reporting the correlation between the occurrence of cutaneous rash and improved clinical outcomes. ${ }^{27}$ For this reason, some authors consider cutaneous rash a useful marker for predicting treatment responses in NSCLC. ${ }^{28}$

\section{Conclusion}

In conclusion, the concurrent combination of erlotinib with RT produced, in our study, a scarce clinical benefit limited to CR and longer CSS rate in the treatment of unresectable or locally advanced NSCLC. Although increased toxicity was associated with combined treatment, it was largely manageable. Erlotinib did not increase the toxicity within the irradiation field. Further studies in molecularly unselected lung cancer patients treated with EGFR TKIs and RT are not indicated in our opinion. Use of predictive biomarkers to identify patients most likely to benefit is essential to continue research in this direction.

\section{Acknowledgment}

The authors would like to thank Itxaso Mora for her help during the collection of clinical data.

\section{Disclosure}

The authors report no conflicts of interest in this work.

\section{References}

1. Ettinger DS, Akerley W, Borghaei H, et al. Non-small cell lung cancer. J Natl Compr Canc Netw. 2012;10(10):1236-1271.

2. Jemal A, Bray F, Center MM, Ferlay J, Ward E, Forman D. Global cancer statistics. CA Cancer J Clin. 2011;61(2):69-90.

3. Goldstraw P, Crowley J, Chansky K, et al. The IASLC Lung Cancer Staging Project: proposals for the revision of the TNM stage groupings in the forthcoming (seventh) edition of the TNM classification of malignant tumours. J Thorac Oncol. 2007;2(8):706-714.
4. Ramalingam S, Belani C. Systemic chemotherapy for advanced nonsmall cell lung cancer: recent advances and future directions. Oncologist. 2008;13(Suppl 1):5-13.

5. Morgensztern D, Ng S, Gao F, Govindan R. Trends in stage distribution for patients with non-small cell lung cancer: a National Cancer Database survey. J Thorac Oncol. 2010;5(1):29-33.

6. Penland SK, Socinski MA. Management of unresectable stage III nonsmall cell lung cancer: the role of combined chemoradiation. Semin Radiat Oncol. 2004;14(4):326-334.

7. Fidler MJ, Kim AW, Zusag T, Bonomi P. Treatment of locally advanced non-small cell lung cancer. Clin Adv Hematol Oncol. 2009;7(7): 455-464, 479-480.

8. Salama JK, Vokes EE. New radiotherapy and chemoradiotherapy approaches for non-small-cell lung cancer. J Clin Oncol. 2013;31(8): 1029-1038.

9. Le Chevalier T, Arriagada R, Quoix E, et al. Radiotherapy alone versus combined chemotherapy and radiotherapy in unresectable non-small cell lung carcinoma. Lung Cancer. 1994;10 (Suppl 1):S239-S244.

10. Pritchard RS, Anthony SP. Chemotherapy plus radiotherapy compared with radiotherapy alone in the treatment of locally advanced, unresectable, non-small-cell lung cancer. A meta-analysis. Ann Intern Med. 1996; 125(9):723-729.

11. Provencio M, Isla D, Sanchez A, Cantos B. Inoperable stage III non-small cell lung cancer: current treatment and role of vinorelbine. J Thorac Dis. 2011;3(3):197-204.

12. Auperin A, Le Pechoux C, Rolland E, et al. Meta-analysis of concomitant versus sequential radiochemotherapy in locally advanced non-small-cell lung cancer. J Clin Oncol. 2010;28(13):2181-2190.

13. O'Rourke N, Roque IFM, Farre Bernado N, Macbeth F. Concurrent chemoradiotherapy in non-small cell lung cancer. Cochrane Database Syst Rev. 2010;6:CD002140.

14. Phernambucq EC, Spoelstra FO, Verbakel WF, et al. Outcomes of concurrent chemoradiotherapy in patients with stage III non-smallcell lung cancer and significant comorbidity. Ann Oncol. 2011;22(1): 132-138.

15. Mok TS. Personalized medicine in lung cancer: what we need to know. Nat Rev Clin Oncol. 2011;8(11):661-668.

16. Pirker R. EGFR-directed monoclonal antibodies in non-small cell lung cancer. Target Oncol. 2013;8(1):47-53.

17. Schmid-Bindert G. Update on antiangiogenic treatment of advanced non-small cell lung cancer (NSCLC). Target Oncol 2013;8(1):15-26.

18. D'Arcangelo M, Cappuzzo F. Erlotinib in the first-line treatment of non-small-cell lung cancer. Expert Rev Anticancer Ther. 2013;13(5): 523-533.

19. Mehta VK. Radiotherapy and erlotinib combined: review of the preclinical and clinical evidence. Front Oncol. 2012;2:31.

20. Casas F, Vinolas N, Sanchez-Reyes A, et al. Spanish patterns of care for 3D radiotherapy in non-small-cell lung cancer. Int J Radiat Oncol Biol Phys. 2006;65(1):138-142.

21. [No authors listed]. Chemotherapy in non-small cell lung cancer: a metaanalysis using updated data on individual patients from 52 randomised clinical trials. Non-small Cell Lung Cancer Collaborative Group. BMJ. 1995;311(7010):899-909.

22. Prados C, Galera R, Santiago A. Patrón pulmonar intersticial unilateral como primera manifestación de una endocarditis bacteriana. [Unilateral interstitial lung pattern as a first sign of a bacterial endocarditis]. Arch Bronconeumol. 2010; 46(4):206-207. Spanish.

23. Li G, Hu W, Wang J, et al. Phase II study of concurrent chemoradiation in combination with erlotinib for locally advanced esophageal carcinoma. Int J Radiat Oncol Biol Phys. 2010;78(5):1407-1412.

24. Iannitti D, Dipetrillo T, Akerman P, et al. Erlotinib and chemoradiation followed by maintenance erlotinib for locally advanced pancreatic cancer: a phase I study. Am J Clin Oncol. 2005;28(6):570-575.

25. Wu YL, Lee JS, Thongprasert $\mathrm{S}$, et al. Intercalated combination of chemotherapy and erlotinib for patients with advanced stage non-smallcell lung cancer (FASTACT-2): a randomised, double-blind trial. Lancet Oncol. 2013;14(8):777-786. 
26. Lonardi F, Coeli M, Pavanato G, Adami F, Gioga G, Campostrini F. Radiotherapy for non-small cell lung cancer in patients aged 75 and over: safety, effectiveness and possible impact on survival. Lung Cancer. 2000;28(1):43-50.

27. Wacker B, Nagrani T, Weinberg J, Witt K, Clark G, Cagnoni PJ. Correlation between development of rash and efficacy in patients treated with the epidermal growth factor receptor tyrosine kinase inhibitor erlotinib in two large phase III studies. Clin Cancer Res. 2007;13(13): 3913-3921.
28. Liu HB, Wu Y, Lv TF, et al. Skin rash could predict the response to EGFR tyrosine kinase inhibitor and the prognosis for patients with non-small cell lung cancer: a systematic review and meta-analysis. PLoS One. 2013;8(1):e55128. 


\section{Supplementary materials}

Table SI Study design, endpoints, and study assessment

\begin{tabular}{|c|c|}
\hline Study type & $\begin{array}{l}\text { Multicenter, randomized, controlled, open-label, prospective, and nationwide Phase II trial } \\
\text { Involved patients with unresectable or locally advanced NSCLC } \\
\text { Randomization was centralized, and patients were stratified according to ECOG-PS (0-I versus } 2 \text { ) and stage of } \\
\text { disease (I-II versus III) }\end{array}$ \\
\hline Criteria for inclusion & $\begin{array}{l}\text { Aged over } 18 \text { years } \\
\text { Histologically confirmed stage IA-IIIB unresectable NSCLC } \\
\text { ECOG-PS } 0-2 \\
\text { Adequate hematologic, hepatic, renal, and respiratory function } \\
\text { No previous CT or RT treatment }\end{array}$ \\
\hline Main exclusion criteria & $\begin{array}{l}\text { Previous CT or RT treatment } \\
\text { Previous treatment with erlotinib or any anti-EGFR } \\
\text { Any concomitant severe disorder or neoplasia } \\
\text { Pregnancy }\end{array}$ \\
\hline Primary endpoint & Feasibility and tolerability pattern of the addition of erlotinib to thoracic RT \\
\hline Secondary endpoint & $\begin{array}{l}\text { Efficacy of the combined treatment: } \\
\text { - Progression-free survival (PFS) } \\
\text { - Time to progression (TTP) } \\
\text { - Time to treatment failure (TTF) } \\
\text { - Overall survival } \\
\text { - Cancer specific survival (CSS) } \\
\text { - Objective response rate (ORR) }\end{array}$ \\
\hline Study arms & $\begin{array}{l}\text { - Control arm (CA): patients receiving } 66 \text { Gy with three-dimensional conformal thoracic RT ( } 5 \text { days/week, } \\
\text { conventional fractionation } 200 \text { cGy/day) } \\
\text { - Experimental arm (EA): patients receiving the same radiotherapy dose scheme but combined with erlotinib } \\
\text { (I50, } 100 \text {, or } 50 \mathrm{mg} \text { po for maximum } 6 \text { months) }\end{array}$ \\
\hline Number of patients & $\begin{array}{l}\text { On the basis of primary endpoint and the lack of information concerning the combination of erlotinib and } \\
\text { radiotherapy, the total number of patients enrolled in the study was } 90 \text {, ie, } 30 \text { in the CA and } 60 \text { in the EA }\end{array}$ \\
\hline Ethics and consent & $\begin{array}{l}\text { All patients gave their informed consent before participating in the study. Procedures were performed in } \\
\text { accordance with the guidelines established by the ethics committee of each participating center and the Declaration } \\
\text { of Helsinki }\end{array}$ \\
\hline
\end{tabular}

Abbreviations: NSCLC, non-small-cell lung cancer; ECOG-PS, Eastern Cooperative Oncology Group Performance Status; EGFR, epidermal growth factor receptor; $\mathrm{RT}$, radiotherapy; $\mathrm{CT}$, chemotherapy; po, per oral administration. 
Table S2 Demographic and clinical characteristics of patients at baseline

\begin{tabular}{|c|c|c|}
\hline Characteristics & RT $\operatorname{arm}(\mathbf{N}=30)$ & $\mathbf{R T}+$ erlotinib arm $(\mathbf{N}=60)$ \\
\hline Sex, male, n (\%) & $30(100.0)$ & $54(90.0)$ \\
\hline Age, median (IQR) & $79.8(74.6-81.2)$ & $79.3(76.7-81.7)$ \\
\hline \multicolumn{3}{|l|}{ Smoking habit, n (\%) } \\
\hline Ex-smokers & $15(50.0)$ & $43(71.7)$ \\
\hline Smokers & 14 (46.7) & $13(21.7)$ \\
\hline Nonsmokers & I (3.3) & $4(6.7)$ \\
\hline History of interstitial lung disease & I (3.3) & $4(6.7)$ \\
\hline \multicolumn{3}{|l|}{ ECOG-PS, n (\%) } \\
\hline 0 & $0(0.0)$ & $3(5.0)$ \\
\hline 1 & $18(60.0)$ & $38(63.3)$ \\
\hline 2 & $12(40.0)$ & $19(31.7)$ \\
\hline \multicolumn{3}{|l|}{ Tumor stage, n (\%) } \\
\hline IA & $4(13.3)$ & $7(11.7)$ \\
\hline IB & $6(20.0)$ & II (18.3) \\
\hline IIA & $\mathrm{I}(3.3)$ & $\mathrm{I}(\mathrm{I} .7)$ \\
\hline IIB & $3(10.0)$ & $7(11.7)$ \\
\hline IIIA & $4(13.3)$ & $10(16.7)$ \\
\hline IIIB & $12(40.0)$ & $23(38.3)$ \\
\hline IV & $0(0.0)$ & $\mathrm{I}(\mathrm{I} .7)$ \\
\hline \multicolumn{3}{|l|}{ Lung tumor histology, n (\%) } \\
\hline Squamous-cell carcinoma & 14 (46.7) & $28(46.7)$ \\
\hline Large-cell carcinoma & II (36.7) & $14(23.3)$ \\
\hline Adenocarcinoma & $4(13.3)$ & $12(20.0)$ \\
\hline Others & I (3.3) & $6(10.0)$ \\
\hline Unresectable tumor, n (\%) & $17(56.7)$ & $31(5 \mid .7)$ \\
\hline
\end{tabular}

Abbreviations: IQR, interquartile range; ECOG-PS, Eastern Cooperative Oncology Group-Performance Status; RT, radiotherapy.

Table S3 Main adverse events related to treatment arms

\begin{tabular}{|c|c|c|c|c|}
\hline \multirow[t]{2}{*}{ Adverse events } & \multicolumn{2}{|l|}{$R T \operatorname{arm}(\mathbf{N}=29)$} & \multicolumn{2}{|c|}{ RT + erlotinib arm $(\mathbf{N}=60)$} \\
\hline & AE grade I-2, n (\%) & AE grade $3-5, n(\%)$ & AE grade I-2, n (\%) & AE grade $3-5, \mathrm{n}(\%)$ \\
\hline \multicolumn{5}{|l|}{ Cutaneous toxicity } \\
\hline Rash/desquamation & $2(6.9)$ & $0(0.0)$ & $34(56.7)$ & $8(13.3)$ \\
\hline Radiation skin injury & 14 (48.3) & I (3.4) & $23(38.3)$ & $2(3.3)$ \\
\hline Dry skin & $0(0.0)$ & $0(0.0)$ & $12(20.0)$ & $\mathrm{I}(\mathrm{I} .7)$ \\
\hline Fatigue & $7(24.1)$ & $0(0.0)$ & $27(45.0)$ & $5(8.3)$ \\
\hline Hyporexia & $4(13.8)$ & I (3.4) & $23(38.3)$ & $5(8.3)$ \\
\hline \multicolumn{5}{|l|}{ Gastrointestinal } \\
\hline Diarrhea & $2(6.9)$ & I (3.4) & $26(43.3)$ & $4(6.7)$ \\
\hline Mucositis & II (37.9) & $0(0.0)$ & $18(30.0)$ & $0(0.0)$ \\
\hline Dysphagia & $5(17.2)$ & $0(0.0)$ & $9(15.0)$ & $0(0.0)$ \\
\hline Constipation & $0(0.0)$ & $0(0.0)$ & II (I8.3) & $0(0.0)$ \\
\hline Vomiting & $0(0.0)$ & I (3.4) & $8(13.3)$ & I (I.7) \\
\hline Infection & $2(6.9)$ & $\mathrm{I}(3.4)$ & $6(10.0)$ & $4(6.7)$ \\
\hline \multicolumn{5}{|l|}{ Pulmonary } \\
\hline Cough & $10(34.5)$ & $0(0.0)$ & $22(36.7)$ & I (I.7) \\
\hline Dyspnea & $6(20.7)$ & $4(13.8)$ & 13 (21.7) & $6(10.0)$ \\
\hline Pneumonitis & $3(10.3)$ & $3(10.3)$ & $9(15.0)$ & $2(3.3)$ \\
\hline \multicolumn{5}{|l|}{ Hematological } \\
\hline Anemia & $3(10.3)$ & $0(0.0)$ & $6(10.0)$ & $2(3.3)$ \\
\hline
\end{tabular}

Note: Main AEs selected occurred in a minimum of ten patients.

Abbreviations: $R T$, radiotherapy; $A E$, adverse event. 


\section{Publish your work in this journal}

OncoTargets and Therapy is an international, peer-reviewed, open access journal focusing on the pathological basis of all cancers, potential targets for therapy and treatment protocols employed to improve the management of cancer patients. The journal also focuses on the impact of management programs and new therapeutic agents and protocols on

patient perspectives such as quality of life, adherence and satisfaction. The manuscript management system is completely online and includes a very quick and fair peer-review system, which is all easy to use. Visit http://www.dovepress.com/testimonials.php to read real quotes from published authors.

Submit your manuscript here: http://www.dovepress.com/oncotargets-and-therapy-journal 